\title{
Understanding the Dynamics of Magnetic Reconnection Layer
}

\author{
Masaaki Yamada
}

Received: 7 February 2011 / Accepted: 17 May 2011 / Published online: 12 August 2011

(C) The Author(s) 2011. This article is published with open access at Springerlink.com

\begin{abstract}
We review progress in understanding the dynamics of a typical magnetic reconnection layer by describing the historical development of theory and the recent findings and discoveries in space and laboratory plasmas. The emphasis is on the dynamics of electrons moving with respect to ions in the collision-free neutral sheet. We make a detailed comparison of experimental results from the Magnetic Reconnection Experiment (MRX) with those from theory and numerical simulations. The collaboration between space and laboratory scientists on reconnection research has recently reached a point where we can compare measurements of the reconnection layer profile in detail with support from numerical simulations. In spite of the large difference in physical scales by $10^{6}-10^{7}$, we find remarkable commonalities in the features of the magnetic reconnection region in laboratory and magnetospheric plasmas. A newly planned laboratory experiment, in which a current sheet is swept in the way a magnetosphere current sheet crosses space satellites, is also described.
\end{abstract}

Keywords Magnetic reconnection · Laboratory astrophysics experiments · Two fluid physics

\section{Introduction}

For over a half century, one of the most important questions on magnetic reconnection has been why reconnection occurs so fast in a conductive plasma in comparison with the rate predicted by classical magnetohydrodynamic (MHD) theory. During the past dozen years, important progress in understanding the physics of fast reconnection has been made through numerical simulations, observations from satellites, and dedicated laboratory plasma experiments (Yamada et al. 2010). It is now recognized that two-fluid effects (Drake and Shay 2007; Birn et al. 2001; Yamada 2007), resulting from the different behavior of ions and electrons, are important within the critical layer where reconnection occurs. In benchmark

M. Yamada $(\bowtie)$

Center of Magnetic Self-organization, Princeton Plasma Physics Laboratory, Princeton University,

Princeton, NJ 08543, USA

e-mail: myamada@pppl.gov 
studies of the two-fluid physics of the local reconnection layer, important progress has been made in understanding fast collisionless reconnection. Hall effects are now considered to facilitate the fast reconnection observed in collisionless neutral sheets in the magnetosphere and in laboratory plasmas.

In this tutorial article, we review the progress of understanding the dynamics of a prototypical magnetic reconnection layer by describing the historical development of theory and the recent findings and discoveries in space and laboratory plasmas. Also we intend to address the relationships between the local physics of the reconnection layer and the external boundary conditions. One of our key questions is how large-scale systems generate local reconnection structures in realistic 3-D geometries through formation of a single or multiple current sheets or magnetic islands.

Magnetic reconnection has been described for a long time through the resistive MHD theory that treats the plasma as a single fluid (Parker 1957; Petschek 1964). The MHD framework is based on the assumption that electrons and ions move together as one fluid even in the presence of internal currents, and is generally regarded as a good approximation to describe the global plasma dynamics. But this condition does not generally hold in a collision-free reconnection layer such as the one created in the magnetosphere where ions and electrons behave differently. Reconnection layers in the magnetopause (Hughes 1995; Mozer et al. 2002; Phan et al. 2007) have thicknesses that are comparable to the ion skin depth $\left(c / \omega_{p i}\right)$. Ions become demagnetized in the collision-free reconnection layer with electrons still magnetized, and the relative drift velocity between electrons and ions can be very large. In this region, the ion skin depth is comparable to the ion gyro-radius and the magnetized electrons generate a strong Hall current. This Hall current, which contributes to the enhanced electric field perpendicular to the reconnection plane, is considered responsible for speeding up the rate of reconnection, and partially, if not completely, provides an answer to one of the oldest questions on reconnection, why reconnection occurs so fast. Further, the two fluid effects can invoke electromagnetic and electrostatic turbulence at high frequency $\left(\omega>\omega_{c i}\right)$ (Shinohara et al. 1998; Bale et al. 2002), which can increase the magnetic reconnection rate.

Let us review the major progress achieved in the past decade:

- It has been observed in a laboratory experiment and numerical simulations that the shape of the reconnection layer changes dramatically as the collisionality of plasma is varied. In a highly collisional plasma, a rectangular-shaped Sweet-Parker reconnection layer is identified with a slow classical reconnection rate. In the collisionless regime, the shape of the reconnection layer changes to a Petschek type double-wedge shape with a much faster reconnection rate, and the electron diffusion region is identified.

- In numerical simulations, dedicated laboratory experiments and space satellite data, Hall effects have been verified by observations of an out-of-reconnection plane quadrupolar structure in the reconnecting magnetic field. This provides experimental evidence for the presence of collisionless two-fluid processes that speed up the reconnection rate.

- In a laboratory experiment, the reconnection rate is found to increase rapidly as the ratio of the electron mean free path to the system size increases. This result is attributed to the two-fluid dynamics at the reconnection layer. Also multiple reconnection layers have been identified both in numerical simulation and the laboratory plasmas in the collisionless regime. They are now considered to generate impulsive reconnection.

- Electrostatic and electromagnetic fluctuations are observed in the neutral sheets of laboratory and space plasmas with notable similarities in their characteristics. Although a correlation was found between the reconnection rate and the amplitude of electromagnetic waves in some laboratory experiments, a causal relationship is yet to be found. 
It is the author's view that magnetic reconnection is influenced and determined both by local plasma dynamics in the reconnection region and global boundary conditions. One major question is how large-scale systems generate local reconnection structures through formation of current sheets either spontaneously or via imposed boundary conditions.

Very recently, two review papers were written by the present author (Yamada et al. 2010; Zweibel and Yamada 2009) to cover both the major experimental results and space observations that have provided useful information on the physics of magnetic reconnection over the past few decades. The present short review is different in emphasis from these recent review papers. Here we describe the evolution of our understanding of the mechanisms of the reconnection layer by comparing theory, numerical simulation results, and recent laboratory and space measurements. Because of limited space, the laboratory data are primarily from MRX (Yamada et al. 1997a, 1997b). Many fine works which have been carried out related to the present subject by other laboratory experiments (Egedal et al. 2001, 2008; Brown et al. 2006) are not covered by this article. More extensive coverage is made by other review books (Biskamp 2000; Birn and Priest 2007) as well as by the two review papers mentioned above.

Recently, collaboration between space observers and laboratory experimentalists has increased significantly. Laboratory physicists can create a prototypical reconnection processes in a controlled manner while space scientists can take very detailed measurements of the particle distribution functions with satellites. Despite the large difference in physical scales, we find remarkable commonalities in the features of the magnetic reconnection region in laboratory and space plasmas. The collaboration has recently reached a point we can compare measurements of the reconnection layer profile in space and laboratory plasmas. We also discuss the possibility of utilizing laboratory experiments to help understand space satellite measurements such as those from the upcoming in MMS (Magnetospheric Multiscale Mission).

\section{Historical Development of Theory and Observations}

\subsection{Resistive MHD Theories}

How do magnetic field lines move around in plasmas and how do they reorganize? The ideal MHD theory was developed in the early 1950's to describe the dynamics of highly conductive plasmas $(\eta=0)$, where magnetic field lines always move with plasma and remain intact with $E_{\|}=\mathbf{E} \cdot \mathbf{B} / B=0$ (Parker 1957; Sweet 1958; Dungey 1995). If we consider magnetic field lines approaching each other in a plasma, magnetic field gradients become locally strong at the meeting point. Plasma flows can lead to singular current density sheets where $E_{\|}$becomes sufficiently large (finite) to induce non-MHD plasma behavior so that a magnetic field line can lose its original identity due to diffusion. Dungey (1953) showed that such a current sheet can indeed be formed by the collapse of magnetic field near an $X$-type neutral point (Fig. 1), and he suggested that "lines of force can be broken and rejoined." When the field lines are reconnected, the topology of magnetic configuration can change and large $\mathbf{j} \times \mathbf{B}$ MHD forces often result.

Sweet and Parker addressed magnetic reconnection problems in a situation where solar coronal fields are merging and transformed the reconnection region into a 2-dimensional reconnection boundary layer in which oppositely directed field lines merge as shown in Fig. 1(b) and Fig. 2. In their model, magnetic fields of opposite polarity approach in the rectangular shaped reconnection region where the incoming field lines merge, and newly 
Fig. 1 Reconnection layer considered by Dungy (a)

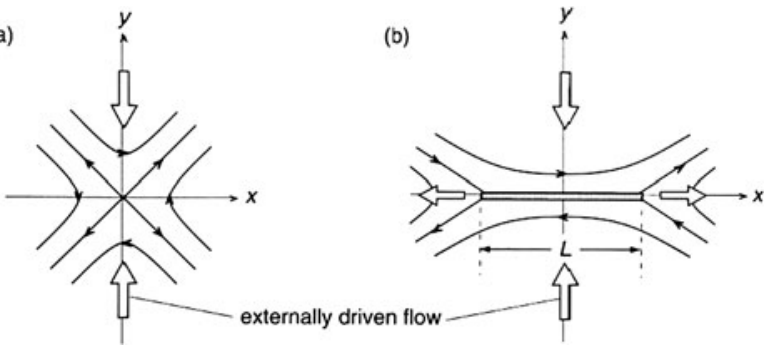

Fig. 2 Sweet-Parker model for analysis of a reconnection layer: Oppositely directed field lines merge in the diffusion region of width $2 \delta$ and length $2 L$

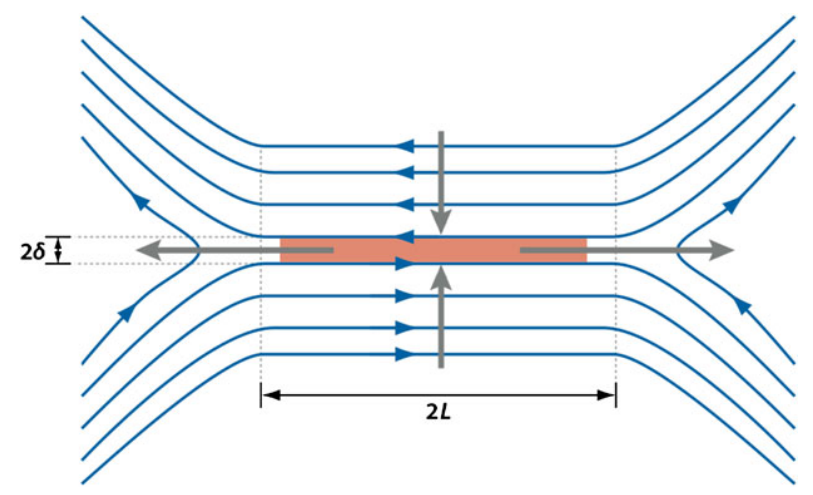

reconnected field lines emerge and move away. During this process, a dissipation of magnetic field occurs due to resistivity. This 2-D MHD model introduced the important concept that the magnetic reconnection rate can be calculated quantitatively through a magnetic flux transfer between two geometrically separated plasma regions, assuming uniformity in the third dimension.

For analysis of the local reconnection layer using resistive MHD formulation, the motion of magnetic field lines in a plasma can be described by combining Ohm's law and Maxwell's equations,

$$
\begin{aligned}
& \mathbf{E}+\mathbf{v} \times \mathbf{B}=\eta \mathbf{j}, \\
& \partial \mathbf{B} / \partial t=-\nabla \times \mathbf{E},
\end{aligned}
$$

to yield

$$
\partial \mathbf{B} / \partial t=\nabla \times(\mathbf{v} \times \mathbf{B})+\left(\eta / \mu_{0}\right) \nabla^{2} \mathbf{B} .
$$

Here, conventional notations are used for local electric field and magnetic field vectors, $\mathbf{E}, \mathbf{B}$, current density, $\mathbf{j}$, and plasma flow velocity, $\mathbf{v}$. When $\eta=0$, magnetic field lines move with the fluid without any dissipation as described by (3). In resistive MHD plasmas, hydromagnetic flows can lead to the formation of a neutral sheet where the plasma flow is reduced to a finite size and the electric field (E) is balanced with $\eta \mathbf{j}$ in (1). In the rectangular diffusion region shown in Fig. 2, the resistivity term becomes sufficiently large that a magnetic field line can diffuse and lose its original identity and reconnect to another field line. In a steady state (3) can be simplified to

$$
V_{\text {in }} B=\left(\eta / \delta \mu_{0}\right) B .
$$

Using the continuity equation,

$$
V_{\text {in }} L=V_{\text {out }} \delta
$$


and pressure balance between the upstream $\left(p \sim B^{2} / 2 \mu_{0}\right)$ and the downstream $\left(p \sim V^{2} / 2\right)$ regions, namely, $V_{\text {out }}=V_{A}$, a very simple formula is derived for reconnection speed $V_{\text {in }}$;

$$
V_{\text {in }} / V_{A}=\delta / L=1 / \sqrt{S},
$$

where $V_{A}$ is Alfvén velocity and $S=\frac{\mu V_{A} L}{\eta}$ is the (non-dimensional) Lundquist number, the ratio of the Ohmic diffusion time to the crossing time of the Alfvén waves.

In this resistive MHD formulation (Parker 1957), magnetic fields diffuse and dissipate in the rectangular reconnection region illustrated in Fig. 2, where incoming plasma flux is balanced with the outgoing flux, satisfying continuity equations for plasma fluid and magnetic flux. The reconnection rate depends on the Lundquist number $S$, which is usually extremely large: $S$ can be $10^{4}-10^{8}$ in laboratory fusion plasmas, $10^{10}-10^{14}$ in solar flares, and $10^{15}-$ $10^{20}$ in the interstellar medium of the Galaxy. The Sweet-Parker reconnection rate derived above is thus far too slow to describe reconnection phenomena. This slowness comes from the assumption that the plasma and magnetic flux have to go through the narrow rectangular neutral sheet with thickness of $\delta=L / \sqrt{S}$ as shown in Fig. 2. At about the same time another important MHD theory was developed by Furth et al. (1963) to quantitatively calculate the growth of resistive tearing mode in a 2-D current sheet for the analysis of tearing instability. But this theory, also based on resistive MHD, predicts even a slower reconnection rate.

In controlled driven experiments in the Magnetic Reconnection Experiment (MRX), the basic physics of magnetic reconnection was quantitatively studied by measuring the evolution of the measured flux contours of the reconnecting field (Yamada et al. 1997a, 1997b). Experiments have been carried out in the double annular plasma setup in which two toroidal plasmas with annular cross section are formed independently around two flux cores as shown in Fig. 3. Each flux core contains poloidal and toroidal field coils to generate plasma discharges in both the private and common flux regions around the cores and to create a variety of magnetic field line merging situations. After an initial set-up period, poloidal field currents in the two flux cores are decreased to generate a reconnection layer where common flux lines are pulled back toward the $X$-point. Typical plasma parameters are $n_{e} \sim 0.1-$ $1 \times 10^{14} \mathrm{~cm}^{-3}, T_{e}=5-15 \mathrm{eV}, B=0.2-1 \mathrm{kG}, S \sim 500-1000$.

In the collisional regime of MRX operation with $\lambda_{m f p}<c / \omega_{p i}$, "the rectangular" profiles of the reconnection region were verified to be very close to the shape Parker predicted, as seen in Fig. 3. The reconnection rate was measured by monitoring the time evolution of the poloidal flux contours shown in Fig. 3 as a function of plasma parameters and compared with the Sweet-Parker model. When the collision frequency is high, the classical Sweet-Parker reconnection rate was measured (Trintchouk et al. 2003; Kuritsyn et al. 2006). It should be noted that this rectangular shape is consistent with the uniform influx of radial inflow with respect to $Z$ in Fig. 3 (Biskamp 2000).

A significant enhancement of the reconnection rate was observed when the collisionality is reduced to satisfy $\lambda_{m f p}>c / \omega_{p i}$. To explain the observed fast reconnection in a variety of plasmas, an anomalous resistivity theory has often been used by employing an ad-hoc enhanced value of the resistivity, $\eta_{\mathrm{eff}}$, in (1). It should be noted that this formulation is sometimes useful in describing the fast reconnection rate by MHD, although there was no theoretical basis developed at that time for imposing uniformly enhanced resistivity from turbulence or other mechanisms. A generalized Sweet-Parker model was developed by employing the measured effective resistivity $\eta_{\text {eff }}$ to quantitatively explain the reconnection rates observed in MRX (Ji et al. 1998, 1999).

Shortly after the Sweet-Parker theory was developed, another model was proposed by Petschek (1964) to resolve the dilemma of the slow reconnection rate through a narrow 


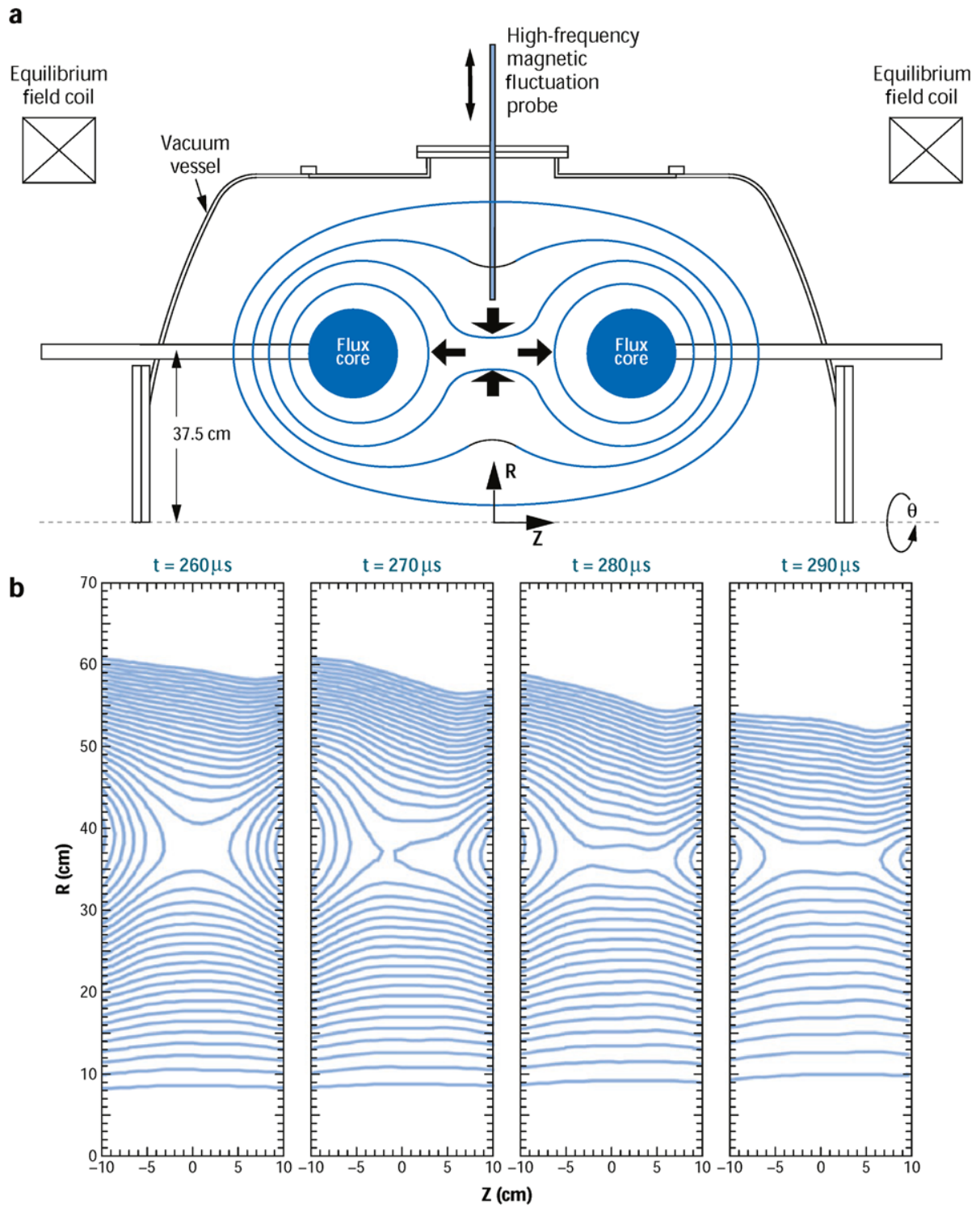

Fig. 3 Illustration of reconnection driven by inductive coils in the MRX apparatus. (b) Time evolution of flux contours during driven reconnection. Magnetic reconnection is demonstrated through measured flux contours. In the low $\beta$ plasma outside the neutral sheet, poloidal flux contours represent magnetic field lines. From Yamada et al. (1997a, 1997b)

reconnection channel by introducing shocks which open up the neutral sheet to a wedge shape, as illustrated in Fig. 4. By eliminating the flow-limiting outgoing current channel as shown in Fig. 4a, this model leads to a much faster rate of reconnection,

$$
V_{\mathrm{Rec}}=\frac{\pi}{8 \ln S} V_{A} \approx(0.1-0.01) V_{A}
$$



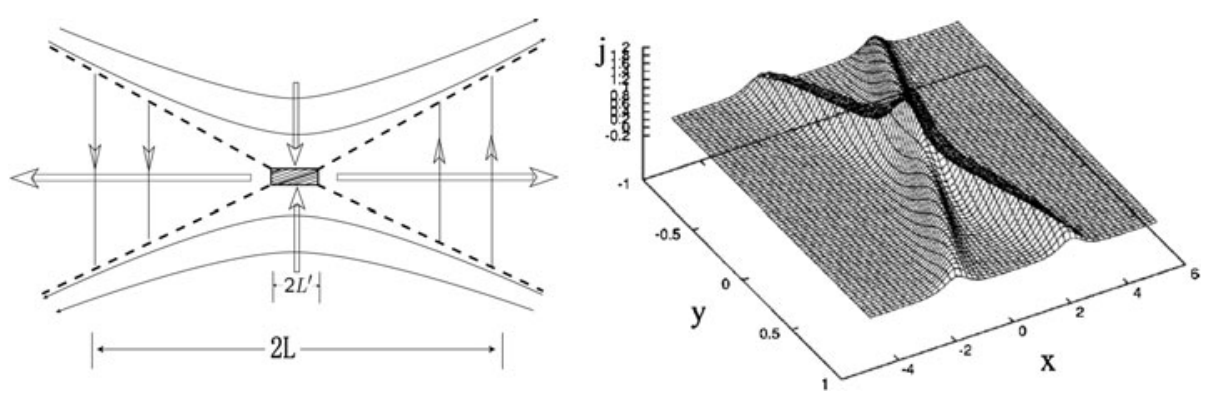

Fig. 4 Left: Petschek reconnection model (Petschek 1964). Right: Current profile from a numerical simulation (Uzdensky and Kulsrud 2000)

While the Petschek reconnection rate is consistent with the observed fast reconnection rates in space and has become very frequently cited, it has not been rigorously established because it is neither compatible with the resistive MHD characteristics nor two-fluid physics mechanisms (Kulsrud 2001; Yamada et al. 2010).

In the past decades, a further analysis of this model has been made employing a locally enhanced resistivity, $\eta_{\text {eff }}(\mathbf{r})$, which is consistent with a notion that a high electron current should induce an anomalous resistivity due to the waves generated by high electron current density at the reconnection region (Sato and Hayashi 1979; Ugai and Tsuda 1977). In this case, (3) has to be modified (Yamada et al. 2010) as,

$$
\partial \mathbf{B} / \partial t=\nabla \times(\mathbf{v} \times \mathbf{B})+\left(\eta_{\mathrm{eff}} / \mu_{0}\right) \nabla^{2} \mathbf{B}+\left(1 / \mu_{0}\right) \nabla \eta_{\mathrm{eff}} \times(\nabla \times \mathbf{B}) .
$$

The locally enhanced resistivity expressed in the second and third terms of the right side increases dissipation near the $X$-point, accelerating electron flows towards $\mathrm{X}$-point. This would generate a wedge shape reconnection region with "slow shocks" formed near the central high resistivity region as shown in Fig. 4b (Uzdensky and Kulsrud 2000). This configuration is free from the constraint of the thin reconnection layer of the Sweet-Parker model and allows for a fast reconnection rate. However, the shock-like structures do not extend all the way to the system size and are thus not consistent with the original wedgeshaped structure of Petschek. We also note that there has been no conclusive experimental evidence to date of shocks observed in association with magnetic reconnection layers in laboratory plasmas.

\subsection{Collisionless Reconnection: Decoupling of Motion Between Electrons and Ions}

The above MHD formulation of the local reconnection layer is based on the assumption that electrons and ions move together as a single fluid even in the presence of internal currents. This framework should be re-evaluated by a realization that this MHD condition does not hold in thin reconnection layers such as those created in the magnetosphere, in which ions become demagnetized and the relative drift velocity between electrons and ions can be very large. Reconnection layers at the magnetopause (Hughes 1995) have thicknesses that are comparable to the ion skin depth $\left(c / \omega_{p i}\right)$. In this region, the ion skin depth is comparable to the ion gyro-radius ( $\beta \sim 1$ ) (Yamada et al. 2000) and only electrons are magnetized, leading to a strong Hall effect. This effect is considered responsible for speeding up the rate of reconnection. Since the two-fluid effects are due to the different behaviors of large orbit ions 
and strongly magnetized electrons with small orbits, electromagnetic or electrostatic turbulence at high frequency $\left(\Omega>\omega_{c i}\right)$ can be excited, which can in turn increase the resistivity and thus the magnetic reconnection rate.

It has been recently recognized that when the Sweet-Parker layer is thinner than the ion skin depth, a two-fluid formulation is necessary to describe the physics of magnetic reconnection. The ratio of the ion skin depth to the Sweet-Parker layer thickness is expressed by

$$
\frac{\left(\frac{c}{\omega_{p i}}\right)}{\delta_{S P}}=\left(\frac{m_{i}}{4 m_{e}}\right)^{1 / 4}\left(\frac{\lambda_{m f p}}{L}\right)^{1 / 2},
$$

where $\lambda_{m f p}$ is the mean free path, and $L$ the global length of the current layer (Yamada et al. 2006; Cassak et al. 2005). Here we have used perpendicular Spitzer resistivity, $\eta_{\perp}=2 \eta_{\|}$. For hydrogen plasmas, this ratio becomes $7\left(\lambda_{m f p} / L\right)^{1 / 2}$ such that the Hall effect becomes dominant when the mean free path is larger than 1/50 of the global length. As the mean free path is increased, the neutral sheet profile changes from a rectangular shape to an $X$ shape with impulsive reconnection features (Yamada 2007). This observation is consistent with the numerical simulation results of Ma and Bhattacharjee (1996).

In the two-fluid formulation, the Ohm's law of (1) should be replaced by the generalized Ohm's law which describes force balance with electron flow, namely,

$$
\mathbf{E}+\mathbf{v} \times \mathbf{B}=\eta_{s} \mathbf{j}+(\mathbf{j} \times \mathbf{B}) / n e+m_{e} / e\left(\partial \mathbf{v}_{e} / \partial t+\mathbf{v}_{e} \cdot \nabla \mathbf{v}_{e}\right)-\nabla \cdot \mathbf{P}_{e} / n e
$$

Here, the conventional notations are again used with electron flow velocity, $\mathbf{v}_{e}$, and variable electron pressure tensor, $\mathbf{P}_{e} ; \mathbf{P} \propto\langle(\mathbf{v}-\langle\mathbf{v}\rangle)(\mathbf{v}-\langle\mathbf{v}\rangle)\rangle$. Generally in (8), all vectors should include fluctuation components and $\eta_{\mathrm{s}}$ denotes the classical Spitzer resistivity based on Coulomb collisions. In a thin reconnection layer in which the magnetized electrons move against demagnetized ions, new effects associated with the Hall term in the generalized Ohm's equation (the 2nd term in the RHS of (8)) contribute to an increased electric field in the direction of sheet current. Equation (8) can be reduced to the ordinary Ohm's law by setting $\mathbf{v}_{e}=\mathbf{v}_{i}=\mathbf{v}$, and by neglecting the electron inertia and pressure tensor terms. It then becomes valid for one-fluid MHD. A large electric field originated from $\mathbf{j} \times \mathbf{B}$ is translated to the fast motion of flux lines in the reconnection plane, or a fast rate of magnetic reconnection. This generalized Ohm's law is equivalent to the equation of motion for electrons,

$$
n m_{e}\left(d \mathbf{v}_{e} / d t\right)=-\nabla \cdot \mathbf{P}_{e}-n e\left(\mathbf{E}+\mathbf{v}_{e} \times \mathbf{B}\right) .
$$

\subsection{Characteristics of Hall Effects}

In the two-fluid formulation, the theoretical treatment of the diffusion region becomes much more complex than that of one-fluid MHD, since magnetized electrons and demagnetized ions flow quite differently. In the past ten years, numerical simulations (Drake and Shay 2007; Horiuchi and Sato 1999; Pritchett 2001; Shay et al. 1998, 2001, 2007; Drake et al. 2006; Daughton et al. 2006; Daughton and Karimabadi 2007; Hesse 2006) of the collision free neutral sheet based on 2-fluid or kinetic codes have been carried out to describe the dynamics of reconnection region. They have demonstrated the importance of the Hall term $[\mathbf{j} \times \mathbf{B}]$ through steady (laminar) cross-field currents of electrons, which contribute to a large apparent resistivity and generate fast reconnection.

Figure 5 shows the results from a particle-in-cell (PIC) simulation by Pritchett (2001). As shown in the figures, the ions, which become demagnetized as they enter the ion diffusion 
Fig. 5 Pattern of ion (top) and electron (bottom) flow vectors obtained by Pritchett 2001. The length scale of ion velocity vectors is amplified by an order of magnitude: Actually, $v_{e} \gg v_{i}$
Ion Flow Velocity

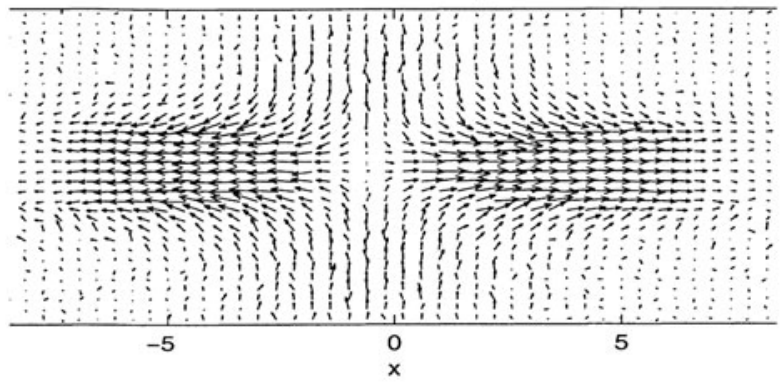

Electron Flow Velocity

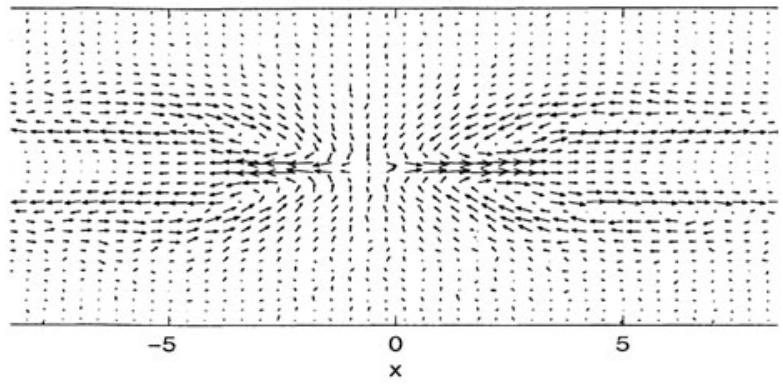

region with the width of $c / \omega_{p i}$, are gradually accelerated while they move across the region, then turn and flow outward to the exit direction. In contrast, the magnetized electrons flow further inward along the separatrices towards the $X$ point. As the electrons' $\mathbf{E} \times \mathbf{B}$ drift motion makes them migrate towards the $X$ line, the magnetic field weakens. The electrons' drift $(E / B)$ becomes larger near the $X$ point and the electrons are ejected out to the exit. This electron flow pattern shown in Fig. 5 generates net circular currents in the reconnection plane and thus creates an out-of-plane magnetic field with a quadrupole profile; this is regarded as a signature of the Hall effect. The increased electric field caused by strong Hall term $[\mathbf{j} \times \mathbf{B}]$ through a steady laminar cross-field current of electrons leads to a fast motion of flux lines $(E=-d \Psi / d t)$ in the reconnection plane, or a fast rate of magnetic reconnection.

Using the three components of the magnetic field vectors measured by a 2-D probe array, precise and conclusive measurements of Hall effects in the neutral sheets were carried out in MRX (Ren et al. 2005; Yamada et al. 2006). Figure 6a shows the contours of the measured out-of-plane quadrupole magnetic field in the diffusion region during magnetic reconnection, together with the vectors of the reconnecting magnetic field in the $R-Z$ plane. The spatial resolution is $4 \mathrm{~cm}$ in the $Z$ direction and is improved to $1 \mathrm{~cm}$ in the $R$ direction by scanning the probe radially and averaging several shots at each position. The quadrupole configuration of the out-of-plane magnetic field $B$ can be clearly seen. The measured amplitude of this quadrupole magnetic field is of order 30-50 G compared with 100-120 G reconnecting field strength.

In Fig. 6b, the reconnecting field lines derived from the three measured components of magnetic fields are shown in 3D. This figure is generated by tracing field lines through magnetic field vectors, $\mathbf{B}$, measured in the $(R, Z)$ plane. The Hall effect in the reconnection region is interpreted as follows. As magnetized electrons are mostly moving along the field lines, they also flow in the out-of-plane direction, being simultaneously accelerated by the reconnecting field $E_{y}$ and pulling the field lines in the $y$ direction. This pulling deforms the 
(a)

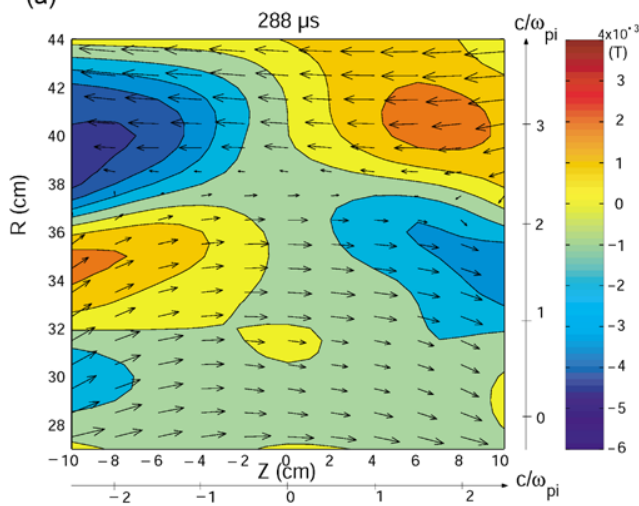

(b)

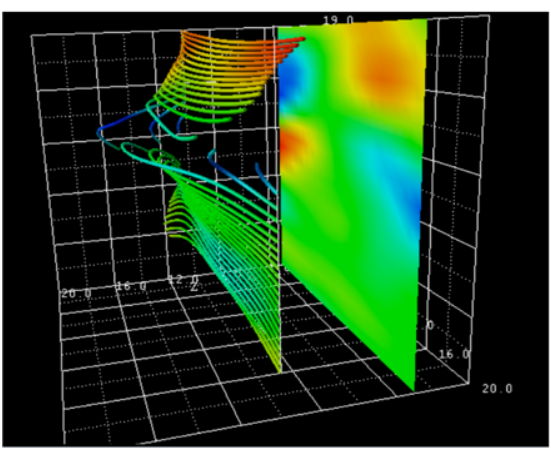

Fig. 6 (a) The magnetic field profiles of the reconnection region for deuterium plasmas. The arrows depict the measured magnetic field vectors in the $R-Z$ plane. The size of the arrows is normalized to the maximum magnetic field strength, $300 \mathrm{G}$. The color coded contour plot shows the out-of-plane magnetic field strength $B_{T}$. (b) 3D profiles of reconnection magnetic field lines measured in MRX. $B_{T}$ is also in color coded contours in the projected back screen

reconnecting field lines to generate the out-of-reconnection plane quadrupole field pattern as shown in Fig. 6 b.

It should be noted, however, that the Hall term alone does not create the energy dissipation necessary for conversion of magnetic energy to particle kinetic energy. It is considered that instead the electron pressure tensor term in (8) and fluctuations can generate energy dissipation particularly at the $X$-point. As reconnection proceeds, the lines of force move into the reconnection current layer, with electrons tied to them. When the electrons reach the $X$-point region, they become demagnetized and diffuse off the field lines causing the lines to break. Then the diffused electrons are accelerated away from $X$-point in both $z$ and $y$ directions, taking energy with them. It has been observed that these electron flows fluctuate on a longer time scale, causing impulsive and turbulent reconnection (Ren et al. 2008). More detailed discussion of electron motions is presented in by Yamada et al. (2010). The laminar flows of electrons are analytically described in the calculation of the Hall effects by Uzdensky and Kulsrud (2006).

Confirmation of the Hall effects has been reported through detection of the out-of-plane magnetic field by others (Mozer et al. 2002; Brown et al. 2006) both in space and laboratory plasmas. The Polar satellite crossed the magnetopause in a southward interplanetary magnetic field (IMF) situation and presented a typical example of the fine structure of the neutral sheet with an out-of-plane magnetic field, a signature of Hall effects.

The in-plane current flows, $\mathbf{V}_{d}=\mathbf{V}_{i}-\mathbf{V}_{e}$, were derived in MRX from the measured out-of-plane Hall field $\left(\mathbf{B}_{t}\right)$ profile from the relation $\mathbf{j}_{\text {in }}=-n e \mathbf{V}_{e}=\operatorname{Curl} \mathbf{B}_{t} / \mu_{0}$ where it is safely assumed $\mathbf{V}_{e} \gg \mathbf{V}_{i}$ (measured as $\sim 0.1 \mathbf{V}_{A}$ ). Figure 7 a shows electron flows in a half $R-Z$ plane derived from the MRX measurements and compared with the simulation result shown in Fig. 7b. In Fig. 7a, the outflow velocity is measured as $V_{d} \sim 2 V_{A}$, verifying that the electron flows represent currents except just at the separatrix regions where the electron and ion flow velocity can be comparable. Figure $7 \mathrm{~b}$ shows, with a remarkable resemblance, the result from a two-fluid simulation in the same physical dimension by adjusting the size with respect to $c / \omega_{p i}$. It shows a very similar pattern of electrons flows: namely, when electrons enter the diffusion region, they flow along the separatrices toward the $X$ point. When they 
pass the separatrices, they make a sharp turn and are accelerated to a value much larger that (ion) Alfvén velocity and flow to the exit directions.

\subsection{Observation of Two-scale Diffusion Layer}

Another important result from the recent comparative study of the reconnection layer between experiments and 2D numerical simulations is the demonstration of a two-scale diffusion layer, in which an electron diffusion layer resides inside the ion diffusion layer whose width is the ion skin depth (Pritchett 2001). In the neutral sheet of MRX, utilizing the same data set that was used for Fig. 7, the electron diffusion region was identified as shown in Fig. 8 and it was found that demagnetized electrons are accelerated to a value that significantly exceeds $V_{A}$ in the outflow direction in the reconnection plane (Ren et al. 2008). The
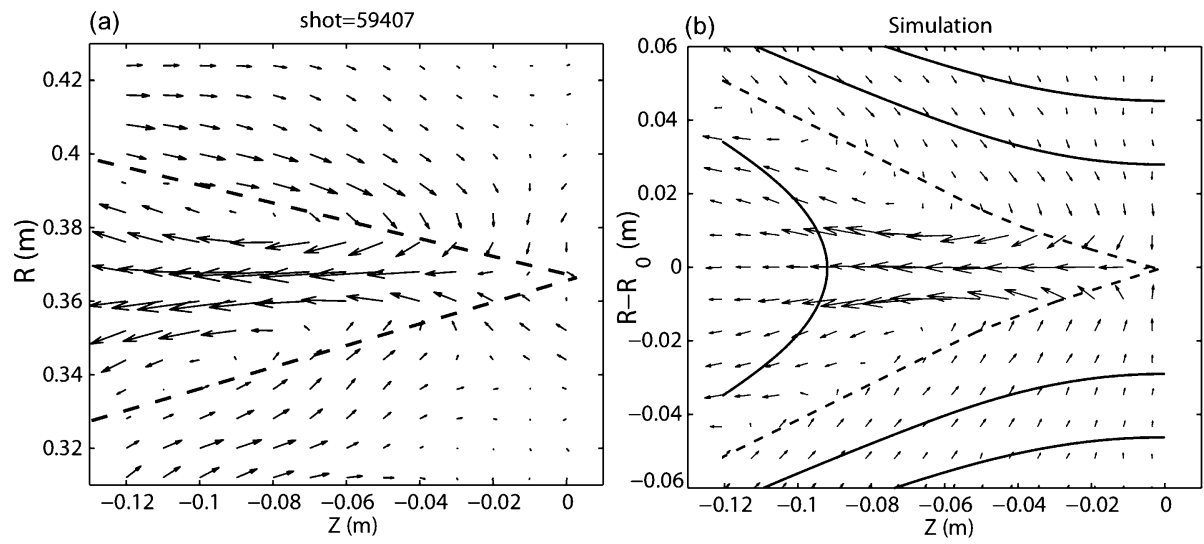

Fig. 7 (a) The electron drift velocity arrows in a half reconnection plane, $\boldsymbol{V}_{d}=\boldsymbol{V}_{e}-\boldsymbol{V}_{i}$, deduced from the out-of-plane field measurement and inferred separatrices (black broken lines) in a hydrogen plasma, fill pressure = 2 mTorr; (b) Simulation: in-plane electron flows (arrows), flux lines (solid lines), and separatrices (broken lines) from the numerical simulation by Breslau and Jardin (2003)

Fig. 8 (a) The radial profiles of the electron outflow velocity, $V_{e Z}$ (magenta asterisks), and ion outflow velocity, $V_{i} Z$ (blue squares), measured in a helium plasma; (b) The 2D profile of the out-of-plane field, $B_{T}$

(color-coded contours), and the in-plane electron flow velocity, $V_{e}$ (black arrows); (c) $V_{e Z}$ and $V_{i Z}$ as a function of $Z$ (Ren et al. 2008) (a)

(b)

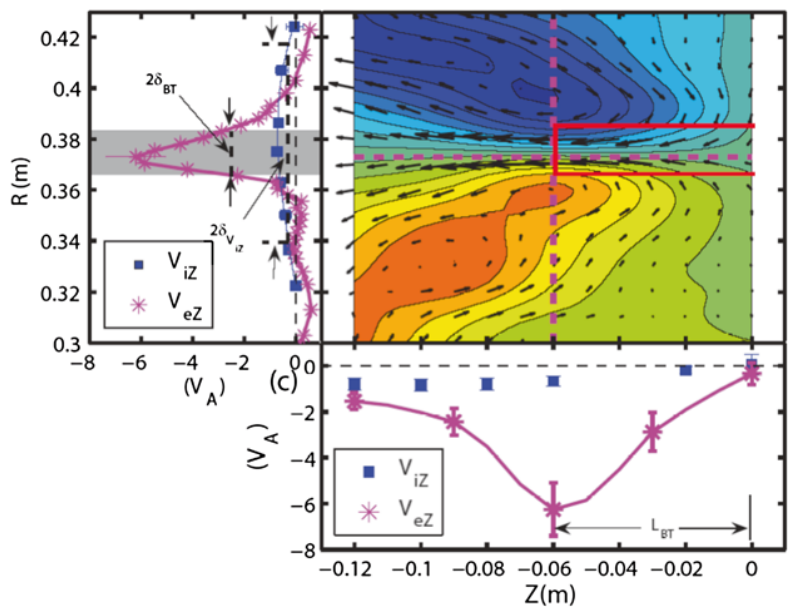


width of the electron diffusion region, which is identified by the profile of the electron outflow, scales with the electron skin depth as $5-7 c / \omega_{p e}$. The electron outflow velocity scales with the local electron Alfvén velocity $\left(\sim 1.2-1.6 V_{e A}\right)$. But the thickness of the electron diffusion layer is $3-5$ times larger than the values $\left(1.5 c / \omega_{p i}\right)$ calculated by 2-D numerical simulations. The effects of collisions have been evaluated to determine how much of the enhancement of the thickness should be attributed to them ( $\mathrm{Ji}$ et al. 2008; Ren et al. 2008; Roytershteyn et al. 2010), and it was concluded they are not a main cause of the discrepancy.

Mozer et al. (2003) and Phan et al. (2007) also identified regimes in the magnetosphere where $\mathbf{j}_{e} \times \mathbf{B}$ is not equal to the local electric field. While they were defined as electron diffusion regimes, the exact location of the observed electron diffusion regime with respect to ion diffusion regime was hard to determine because of the nature of satellite measurement.

The ion outflow channel is shown to be much broader than the electron channel and the ion outflow velocity reaches its maximum value of $V_{A}$ at the exit region, also consistent with the numerical simulations. This electron outflow often occurs impulsively, generating high amplitude magnetic fluctuations at the center of electron diffusion region. The concentration of magnetic fluctuations at the center of current sheet suggests that these fluctuations are excited by the super-Alfvénic electron flows. We note that even with presence of the thin electron diffusion region, the reconnection rate is still primarily determined by the Hall electric field as predicted by the GEM project (Birn et al. 2001). A more comprehensive study is required to determine how the profiles of the electron diffusion layer affect overall reconnection dynamics including energy dissipation.

\subsection{Potential Well in the Electron Diffusion Region and Ion Heating}

As mentioned earlier, although magnetized electrons are mostly moving along the field lines as shown Fig. 6b, they are also simultaneously accelerated by the reconnecting field $E_{y}$, pulling the field lines in the $y$ direction. The dynamics of steady electron flow in the $y$ direction should generate a radial potential drop towards the $X$ region, generating a potential well (w.r.t. $R$ ), since the electrons convey the potential of the upstream points with their large electric conductivity. Measurements by the CLUSTER spacecraft (Wygant et al. 2005) revealed a very thin potential well, with a half width in the range of $60-100 \mathrm{~km}\left[(3-5) c / \omega_{p e}\right]$, around the center of reconnection. Recently a strong potential well has been detected in MRX (Yoo et al. 2011). These observations support the above description of electron dynamics during reconnection very well. Wygant et al. (2005) proposed that the strong radial electric field due to this potential well would trap and heat incoming ions. This is a very plausible argument to explain the high ion temperature observed in space. It should be also noted that the electron current channel can become unstable due to a sharp radial gradient of current density and can make the flux transfer rate fluctuate and generate impulsive reconnection.

\section{Outstanding Problems Regarding Reconnection Layer Dynamics}

Even with the recent notable advances in the two-fluid analysis of the reconnection layer, many important questions remain unsolved. In particular: What really determines the rate of energy conversion from magnetic to kinetic? The Hall term does not provide energy dissipation or break field lines. It was considered that the other terms in the generalized Ohm's law, the inertia term, the pressure tensor term $(\boldsymbol{\nabla} \bullet \mathbf{P})$ in $(8)$, and fluctuations must be responsible for line breaking and the energy conversion. PIC simulation studies (Hesse et al. 1999, 
2004) found that energy dissipation occurs in a small central region of the neutral sheet without a guide field, leading to a relatively small rate of conversion from magnetic energy to particle kinetic energy. This energy conversion rate is too small to explain the observed particle heating during reconnection observed in reversed-field-pinch (RFP) plasma relaxation events (Den Hartog et al. 2007), in spheromak merging experiments (Yamada et al. 1990; Ono et al. 1996), or in solar flare evolution (Lin et al. 2003; Lin 2006). It may be necessary to develop models which would explain the fast conversion rate of magnetic energy, possibly with a large number of reconnection layers. The idea of ion trapping and heating in the potential well at the $X$-region needs further investigation.

As described above, the electron diffusion region has been identified in laboratory and magnetospheric plasmas. The thickness of the observed diffusion region is notably larger than the value $\left(\sim c / \omega_{p e}\right)$ predicted by recent 2-D numerical calculations (Daughton et al. 2004). The reason for this discrepancy is being studied, including the effect of 3-D fluctuations (Daughton et al. 2004) in the vicinity of the electron diffusion layer. At the moment, there is no comprehensive theory that deals with macro- and micro-fluctuations, concerning how they are excited, and how they determine the reconnection rate by influencing the energy conversion processes. Do these fluctuations create anomalous resistivity? The effects of the anomalous viscosity are considered (Drake et al. 2010). To understand these key issues, we need to investigate the relationship between the local reconnection layer dynamics and global magnetic reconnection phenomena.

As mentioned above, if a fast influx of magnetic field lines is imposed into a slow reconnection layer, a sharp negative potential build-up should occur due to the pile up of electrons. The electron current channel can become unstable due to a sharp radial gradient in the current density and the current channel would disrupt. This makes the flux transfer rate fluctuate and generates impulsive reconnection. This was reported by Y. Ren in 2007 and is being studied in more detail on MRX (Dorfman et al. to be published). Analytic theory together with numerical calculations, will aid progress in understanding how fluctuations are excited and how they dissipate energy in the reconnection layer.

\subsection{Multiple Reconnection Layers in a Large System}

Laboratory fusion plasmas and astrophysical systems are generally much larger than the key microphysical scales such as the ion skin depth and ion gyro-radius. Most of the work on reconnection in the past, both numerical and experimental, has investigated relatively small systems - 10-100 ion skin depths. In much larger systems, however, it is found that multiple current sheets or reconnection layers develop in the reconnection region that can affect the reconnection rate in both collisional and collisionless regimes. For example, in simple 2-D resistive MHD simulations for a large plasma with significant Lundquist number $\left(S>10^{4}\right)$, a laminar Sweet-Parker layer is transformed to a chain of secondary magnetic islands and the reconnection process becomes inherently non-steady. The studies by Loreiro et al. (2007) and Bhattajarjee et al. (2009) represent such examples.

The appearance of multiple layers would become dominant particularly in 3-D systems. This process can invoke turbulence in the layer, and new approaches are required to properly describe this turbulent layer. This type of multilayer reconnection can occur in solar flares as well as in fusion plasmas. Recently, it was found that MHD turbulence can enhance the reconnection rate significantly (Lazarian and Opher 2009).

Daughton et al. (2009) have recently found that a collisionless reconnection layer beaks up into many islands and current layers, generating a highly turbulent reconnection region even in their $2 \mathrm{D}$ simulations. This study has been extended to $3 \mathrm{D}$ to discover the features 
Fig. 9 (Top) A 3D VPIC collisional reconnection simulation of MRX uses two flux cores; like the experiment, reconnection is driven by reducing the flux core currents. (Bottom) A 3D VPIC simulation of reconnection layers relevant to Earth's magnetosphere. Open boundary conditions allow plasma and magnetic flux to enter and exit the system. Shown is a density isosurface colored by the ion outflow velocity along magnetic field lines (Daughton et al. 2009)

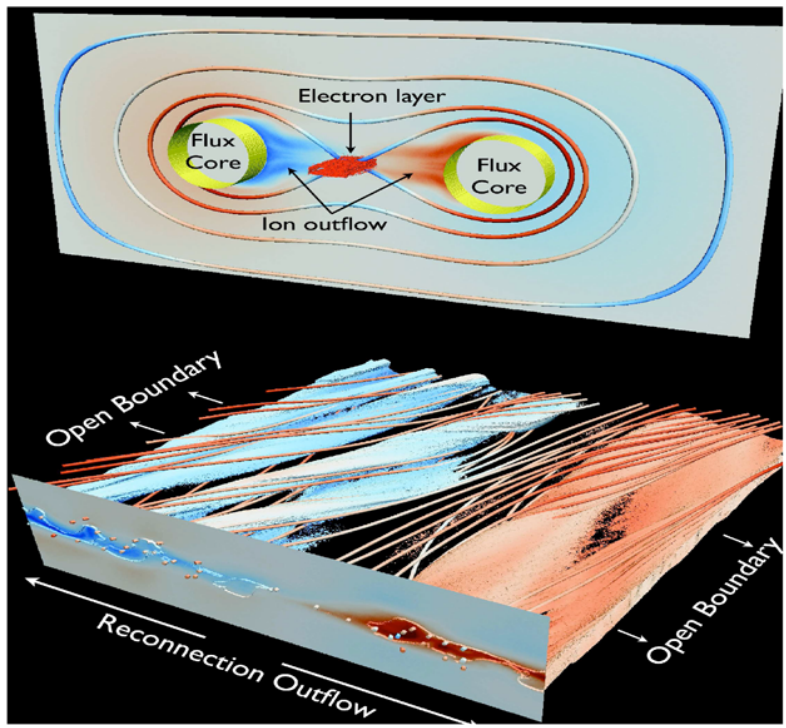

of break-up in the experimental set up of MRX. This result suggests that turbulence can significantly broaden the electron diffusion regime as well as the ion diffusion region.

Figure 9 (top) shows the flux cores, magnetic field structure, ion outflows and electron current sheet from the recent 3D numerical simulation (Daughton et al. 2009) of the MRX experiment. These simulations employ the open boundary model described above with plasma inflows in the boundary. Figure 9 (bottom) shows magnetic island formation along the resonant surfaces across the initial current sheet. In 3D, these islands form extended current sheets or flux ropes at oblique angles above and below the reconnection layer. Over longer time scales, these flux ropes coalesce and intertwine in complex ways to produce new, highly elongated current sheets, which are also unstable to new magnetic island (flux rope) formation. This situation can cause high resistivity and thus fast reconnection, as mentioned earlier. Motion of flux ropes would induce impulsive reconnection. On longer time scales, a generalized Sweet-Parker model with enhanced resistivity and viscosity may be able to describe this fast reconnection.

\subsection{Effects of Boundary Conditions}

The Sweet-Parker solution is considered to depend only on local plasma conditions in the vicinity of the layer. However, with a neutral sheet limiting mass outflow, the reconnection rate in the Sweet-Parker theory is closely coupled to the global boundary condition that determines the length of the layer. In the Petschek model, the length of the Sweet-Parker layer is made smaller than the system size, generating a faster reconnection rate. Priest and Forbes (1986) found that the boundary conditions and resulting global magnetic field structure can drastically change the global reconnection rate. In MRX the large downstream pressure was found (Ji et al. 1999) to slow the outflow and the reconnection rate, demonstrating the importance of boundary conditions. It was found that with the same plasma parameters the reconnection rate decreases with increasing distance between flux cores or equivalently with the system size (Kuritsyn et al. 2007). The reduced reconnection rates in larger systems were attributed to longer current sheets. In addition to this dependence on the system size, the 
current sheet length $L$ was found to depend on the effective resistivity, $\eta_{\mathrm{eff}}$. The exact cause of the anomalous resistivity was not determined at that time. For a given system size, the current sheet length anti-correlates with the effective resistivity; namely, the current sheet length varies inversely with resistivity.

Recently, the effects of external forcing on driven reconnection has been studied in the MRX device. A simple model based on a feedback loop has been developed to explain the details of the linear and overdriven scaling regimes. It is found that driven magnetic reconnection may be modeled as the interplay between the external forcing and the dynamics of the current sheet region. By investigating the effects of external forcing in MRX, linear and overdriven regimes are identified. When the external forcing is applied at a slower rate than the flux penetration timescale of the current layer, the reconnection speed is proportional to the external driving rate; thus, the linear regime. By contrast, in the overdriven regime, the incoming magnetic flux cannot penetrate into the reconnection layer at the rate prescribed by the coils. As a result, the reconnection rate saturates at a value determined by the penetration timescale, namely the intrinsic reconnection speed determined by the local dynamics (Dorfman et al. 2008).

As described in the previous section, the recent simulations with larger system size and open boundary conditions have demonstrated that very long reconnection layers become unstable to secondary islands and significantly increase the reconnection rate (Carter et al. 2001). These results suggest the importance of the interplay between local dissipation and global boundary conditions during magnetic reconnection.

\section{Laboratory Experiments for MMS Satellite Missions}

The MMS (Magnetospheric Multiscale Mission) is the first space mission to be able to explore the dynamics of the reconnection layer down to spatial scales of the order of the electron inertial length $\left(c / \omega_{p e}\right)$. At times, the four spacecraft will be placed in a tetrahedron only $10 \mathrm{~km}$ apart, the expected value of $c / \omega_{p e}$. To resolve these structures, which move past the spacecraft at speeds of order $100 \mathrm{~km} / \mathrm{s}$, requires field measurements down to a $1 \mathrm{~ms}$ cadence and particle distribution functions at $20 \mathrm{~ms}$, an entirely new resolution for particles. The MMS mission has identified interdisciplinary scientists who are working with the experimental team, using past space data and modern simulations, primarily kinetic simulations, to broaden the research scope.

A research program is planned on MRX to utilize laboratory plasma experiments to help design an effective data gathering program for the MMS mission and, more broadly, to achieve a fundamental understanding of magnetic reconnection layer physics. One of the main objectives of the proposed program is to help design optimum MMS spacecraft separations, orbits, data collection strategies, and instrument capabilities before and after the MMS launch, based on laboratory experiments which utilize mock-up four-probe assemblies in well-documented reconnection layer geometries. Experimental measurements combined with numerical computation will provide new insights into aspects of collisionless reconnection, such as the microscopic spatial structures of the electron diffusion region mentioned in this paper and of the ion diffusion region, electrostatic and electromagnetic fluctuations, particle acceleration and heating, and the effect of guide fields of varying strengths.

A preliminary experiment was carried out to test our concept by jogging (sweeping in a controlled speed) the MRX current sheet with a controlled speed of $1-2 \times 10^{5} \mathrm{~cm} / \mathrm{s}$ with a set of probes inserted in a fixed position in the geometry shown in Fig. 3. The advantage of this laboratory experiment is that a simulated data set can be obtained just as the current sheet 
Fig. 10 The radial position of the neutral sheet (defined here as the location with $B_{z}=0$ ) versus time with dotted lines showing the sheet half-width (top) and the radial electric field, $E_{R}$ (bottom) measured throughout the electron diffusion region crossing.

Although this result needs more verification work, it demonstrates a potential well at the electron diffusion region

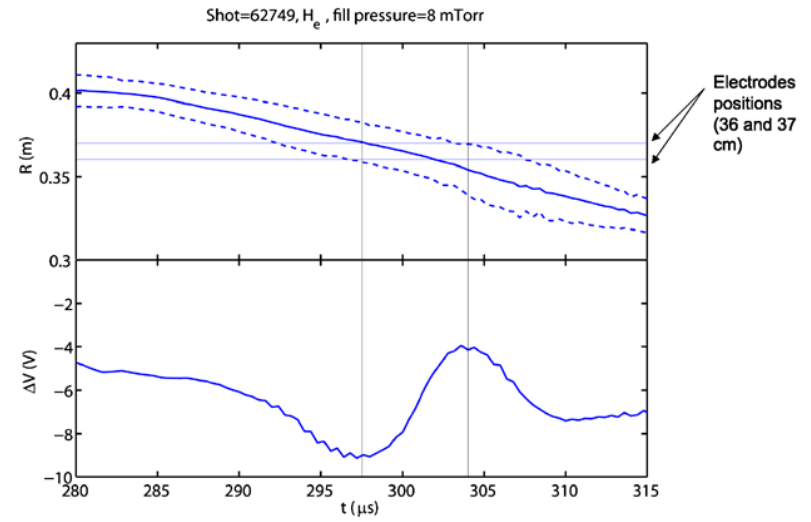

MRX measurement

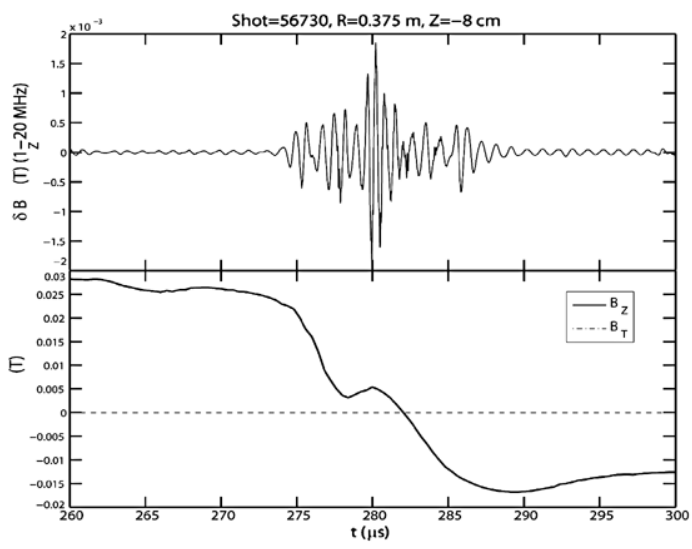

Bale et al., 2004

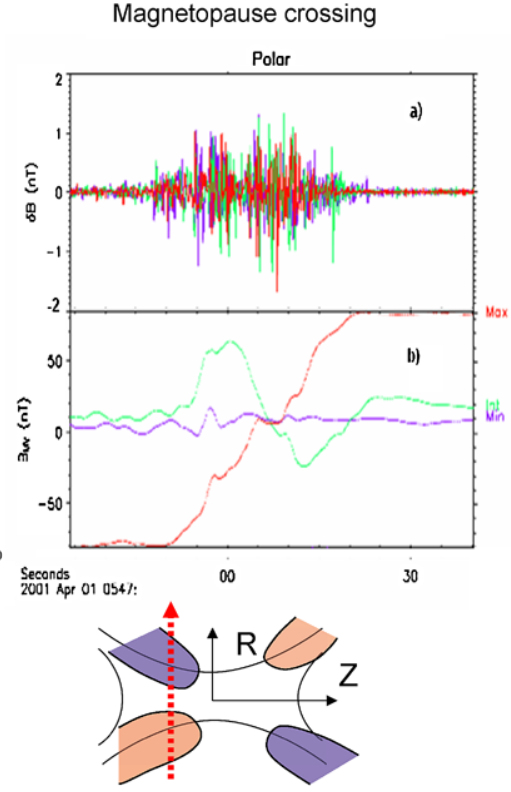

Fig. 11 MRX measurement (left) in which the reconnecting field with corresponding fluctuations (top); and (right) a similar reconnection crossing event in space (bottom right; red is the reconnection field, green is the out-of-plane Hall field) with corresponding fluctuations

region is swept through a probe (satellite) with predetermined velocity and angle. Figure 10 shows a data set from the preliminary sheet-sweeping experiments. Here, the radial position of the neutral sheet versus time has been measured, together with the measured radial electric field. As the sheet sweeps across the probes, a bipolar electric field similar to past space observations (Wygant et al. 2005) can be seen.

Figure 11 depicts the measured time evolution of fluctuating $B$ field $(\delta B)$ and the reconnecting field in MRX in the left column, and corresponding fluctuations and field configurations observed in space in the right column. MRX has also detected an out-of-plane Hall field in a similar shot. Although the detailed comparison has not been yet made, a remarkable resemblance can be seen despite a large difference of scales. High-frequency electrostatic 
and electromagnetic fluctuations have been detected in the reconnecting current sheets in both space (Bale et al. 2002; Mozer et al. 2003) and the laboratory (Carter et al. 2001; Ji et al. 2004).

In agreement with the numerical predictions, it was found that electrostatic fluctuations peak at the low beta edge of the current sheet (Carter et al. 2001), while the electromagnetic fluctuations peak at the current sheet center (Ji et al. 2004; Bale et al. 2002), as seen in Fig. 11. Detailed fluctuation analysis may provide local information about the relative global position of a measurement. By measuring the phase shift in the wave between different spatial locations, the phase velocity can be determined, and thus the dispersion relation of the waves can be uniquely measured. Furthermore, if a wave displays a packet feature, the effective group velocity can be determined.

This type of work would maintain communication and collaboration between experimentalists and observers, supported by numerical simulations. Even beyond the MMS mission, it is in the interest of the broader space and laboratory science communities to nurture and maintain the cross-cutting U.S. basic research.

\section{Summary}

We have reviewed the progress in understanding the dynamics of a typical magnetic reconnection layer by describing the historical development of theory and the recent findings and observations in space and laboratory plasmas. In the collision-free reconnection layer such as seen the magnetosphere, the reconnection layer becomes comparable to the ion skin depth $\left(c / \omega_{p i}\right)$ and ions become demagnetized with electrons still magnetized. The relative flows of electrons against ions in the reconnection plane can generate a strong $\mathbf{j}_{e} \times \mathbf{B}$ force due to the Hall effect. This Hall current, which contributes to the enhanced electric field perpendicular to the reconnection plane, is considered responsible for speeding up the rate of reconnection, providing a partial answer to a very important question why reconnection occurs so fast. But the Hall term does not provide energy dissipation or break field lines. It is considered that the other terms in the generalized Ohm's law, the inertia term, the pressure tensor term $(\nabla \cdot \mathbf{P})$, and fluctuations are responsible for line breaking and the energy conversion.

In dedicated laboratory experiments and space satellite data, Hall effects have been verified by observations of an out-of-reconnection plane quadrupolar structure in the reconnecting magnetic field. This provides experimental evidence for the presence of collisionless two-fluid processes that speed up the reconnection rate. In a laboratory experiment, the reconnection rate is found to increase rapidly as the ratio of the electron mean free path to the system size increases. This result is attributed to the two-fluid dynamics at the reconnection layer. Also multiple reconnection layers have been identified in both numerical simulation and laboratory plasmas in the collisionless regime. They are now considered to induce impulsive reconnection.

It is found that in a single reconnection layer, energy dissipation occurs only in a small central region of the neutral sheet without guide field, leading to a relatively small rate of energy conversion from magnetic to particle kinetic energy. This energy conversion rate is too small to explain the observed particle heating during reconnection observed in space and laboratory plasmas. Recently, 2D and 3D numerical simulations have found that a collisionless reconnection layer breaks up into many islands and current layers, generating a highly turbulent reconnection region causing fast reconnection impulsively. It is suggested that turbulence and waves can significantly broaden the electron diffusion as well as ion diffusion. 
We have also considered impulsive reconnection in the reconnection layer. In the local analysis of collisionless reconnection, the two-fluid dynamics lead to the formation of a narrow electron current layer. Reconnection can be triggered and driven by a spontaneous instability that is excited in the reconnection layer (seen in the MRX reconnection layer) or that is driven by global evolution of plasma profiles (seen in tokamak and other fusion devices). Thus reconnection can often be unsteady or impulsive unless the flux inflow matches the reconnection rate at the reconnection layer.

Thanks to the recent advancement in computer technologies, space and laboratory diagnostic systems, we have a great opportunity to obtain rapid progress in understanding reconnection layer dynamics. The collaboration between space and laboratory scientists has recently reached a point where we can compare measurements of the reconnection layer profile in detail with support from numerical simulations. By carefully comparing results from simulation, space satellites and laboratory experiments, we can achieve a real progress in magnetic reconnection physics. For this purpose, we advocate a new generation of magnetic reconnection experiments on a large-scale device (Yamada and Ji 2010; Ji and Daughton 2011) that will enable investigation of multiple reconnection layers and the physics of energy conversion from magnetic to particles simultaneously.

Acknowledgements The author appreciates a critical reading of this manuscript by Russell Kulsrud and collaboration with the MRX team and the members of Center for Magnetic Self-Organization.

Open Access This article is distributed under the terms of the Creative Commons Attribution Noncommercial License which permits any noncommercial use, distribution, and reproduction in any medium, provided the original author(s) and source are credited.

\section{References}

S. Bale, F. Mozer, T. Phan, Geophys. Res. Lett. 29, 2180 (2002)

A. Bhattajarjee et al., Phys. Plasmas 16, 112102 (2009)

J. Birn, E. Priest (eds.), Reconnection of Magnetic Fields: MHD and Collisionless Theory and Observations (Cambridge Univ. Press, Cambridge, 2007)

J. Birn, J. Drake, M. Shay et al., J. Geophys. Res. 106, 3715 (2001)

D. Biskamp, Magnetic Reconnection in Plasmas (Cambridge Univ. Press, Cambridge, 2000)

J.A. Breslau, S.C. Jardin, Phys. Plasmas 10, 1291 (2003)

M.R. Brown et al., Phys. Plasmas 13, 056503 (2006)

T. Carter et al., Phys. Rev. Lett. 88, 015001 (2001)

P.A. Cassak, M.A. Shay, J.F. Drake, Phys. Rev. Lett. 95, 235002 (2005)

W. Daughton, H. Karimabadi, Phys. Plasmas 14, 072303 (2007)

W. Daughton, G. Lapenta, P. Ricci, Phys. Rev. Lett. 93, 105004 (2004)

W. Daughton et al., Phys. Plasmas 13, 072103 (2006)

Daughton et al., IP Conf. 180, 12055 (2009)

D.J. Den Hartog et al., Nucl. Fusion 47, L17 (2007)

Dorfman et al., Phys. Plasmas 15, 102107 (2008)

J.F. Drake et al., Nature (London) 443, 553 (2006)

J. Drake, M. Shay, in Reconnection of Magnetic Fields: MHD and Collisionless Theory and Observations, ed. by J. Birn, E.R. Priest (Cambridge Univ. Press, Cambridge, 2007), p. 87

J.F. Drake et al., Bull. Am. Phys. Soc. (2010, to be published)

J. Dungey, Philos. Mag. 44, 725 (1953)

J. Dungey, in Physics of the Magnetopause, ed. by P. Song, B. Sonnerup, M. Thomsen. AGU Monograph, vol. 90 (AGU, Washington, 1995), p. 81

J. Egedal et al., Phys. Plasmas 8, 1935 (2001)

J. Egedal et al., J. Geophys. Res. 113, A12207 (2008)

H.P. Furth, J. Killeen, M. Rosenbluth, Phys. Fluids 6, 459 (1963)

M. Hesse, Phys. Plasmas 13, 122107 (2006)

M. Hesse et al., Phys. Plasmas 6, 1781 (1999) 
M. Hesse et al., Phys. Plasmas 11, 5387 (2004)

R. Horiuchi, T. Sato, Phys. Plasmas 6, 4565 (1999)

W. Hughes, in Introduction to Space Physics, ed. by M. Kivelson, C. Russell (Cambridge University Press, London, 1995), p. 227

H. Ji, W. Daughton (2011, to be published)

H. Ji et al., Phys. Rev. Lett. 80, 3256 (1998)

H. Ji et al., Phys. Plasmas 6, 1743 (1999)

H. Ji et al., Phys. Rev. Lett. 92, 115001 (2004)

H. Ji et al., Geophys. Res. Lett. 35, L13106 (2008)

R. Kulsrud, Earth Planets Space 53, 417 (2001)

A. Kuritsyn et al., Phys. Plasmas 13, 055703 (2006)

A. Kuritsyn et al., Geophys. Res. Lett. 34, L16106 (2007)

A. Lazarian, M. Opher, Astrophys. J. 703, 8 (2009)

R.P. Lin, Space Sci. Rev. 124, 233 (2006)

R.P. Lin et al., Astrophys. J. Lett. 595, L69 (2003)

N.F. Loreiro et al., Mon. Not. R. Astron. Soc. 399, L146 (2007)

Z.W. Ma, A. Bhattacharjee, Geophys. Res. Lett. 23, 1673 (1996)

F.S. Mozer, S. Bale, T.D. Phan, Phys. Rev. Lett. 89, 015002 (2002)

F. Mozer et al., Phys. Rev. Lett. 91, 245002 (2003)

Y. Ono et al., Phys. Rev. Lett. 76, 3328 (1996)

E. Parker, J. Geophys. Res. 62, 509 (1957)

H. Petschek, in Proceedings of the AAS-NASA Symposium on the Physics of Solar Flares. NASA Spec. Pub., vol. 50 (NASA, Washington, 1964), p. 425

Phan et al., Phys. Rev. Lett. 99, 255002 (2007)

E. Priest, T. Forbes, J. Geophys. Res. 91, 5579 (1986)

P.L. Pritchett, J. Geophys. Res. 106, 3783 (2001)

Y. Ren et al., Phys. Rev. Lett. 95, 055003 (2005)

Y. Ren et al., Phys. Rev. Lett. 101, 085003 (2008)

V. Roytershteyn et al., Phys. Plasmas 17, 055706 (2010)

T. Sato, T. Hayashi, Phys. Fluids 22, 1189 (1979)

M. Shay, et al., J. Geophys. Res. 103, 9165 (1998)

M.A. Shay, J.F. Drake, M. Swisdak, Phys. Rev. Lett. 99, 155002 (2007)

I. Shinohara et al., J. Geophys. Res. 103, 20365 (1998)

P. Sweet, in Electromagnetic Phenomena in Cosmical Physics, ed. by B. Lehnert (Cambridge University Press, New York, 1958), p. 123

F. Trintchouk, M. Yamada et al., Phys. Plasmas 10, 319 (2003)

M. Ugai, T. Tsuda, J. Plasma Phys. 17, 337 (1977)

D. Uzdensky, R. Kulsrud, Phys. Plasmas 7, 4018 (2000)

D. Uzdensky, R. Kulsrud, Phys. Plasmas 13, 062305 (2006)

J. Wygant et al., J. Geophys. Res. 110, A09206 (2005)

M. Yamada, Phys. Plasmas 14, 058102 (2007)

M. Yamada, H. Ji, in Proc. IAU Meeting on Plasma Astrophys., Italy (2010)

M. Yamada et al., Phys. Rev. Lett. 65, 721 (1990)

M. Yamada et al., Phys. Plasmas 4, 1936 (1997a)

M. Yamada et al., Phys. Rev. Lett. 78, 3117 (1997b)

M. Yamada et al., Phys. Plasmas 7, 1781 (2000)

M. Yamada et al., Phys. Plasmas 13, 052119 (2006)

M. Yamada, R. Kulsrud, H. Ji, Rev. Mod. Phys. 82, 603 (2010)

J. Yoo et al., (2011, to be published)

E. Zweibel, M. Yamada, Annu. Rev. Astr. Astrophys. ANRV 385-AR47-08, p. 291 (2009) 